\title{
Totally Laparoscopic ALPPS: Bilobar Procedure with Preservation of the S3 Portobiliary Triad
}

\author{
Alexander Ferko, MD, PhD ${ }^{1}$, Martin Vojtko, $\mathrm{MD}^{\mathbf{1}}$, Marek Adámik, MD, $\mathrm{PhD}^{\mathbf{1}}$, Ludovít Laca, MD, PhD ${ }^{\mathbf{1}}$, \\ Dagmar Sudeková, $\mathrm{MD}^{2}$, Dagmar Šuteková, $\mathrm{MD}^{3}$, and Marek Smolár, MD, PhD ${ }^{1}$ \\ ${ }^{1}$ Department of Surgery and Transplant Centre, Jessenius Medical Faculty Martin, University Hospital Martin, Comenius \\ University in Bratislava, Bratislava, Slovakia; ${ }^{2}$ Department of Oncology, Teaching Hospital Žilina, Žilina, Slovakia; \\ ${ }^{3}$ Department of Oncology, Jessenius Medical Faculty Martin, University Hospital Martin, Comenius University in \\ Bratislava, Bratislava, Slovakia
}

\begin{abstract}
Background. A laparoscopic approach for associating liver partition and portal vein ligation for staged hepatectomy (ALPPS) would have the potential to decrease morbidity and mortality rates, ${ }^{1}$ as similarly observed with laparoscopic liver surgery. ${ }^{2}$

Methods. A 54-year-old woman with stage IV rectal cancer (cT3dN1M1) was indicated for the 'liver-first' approach. The patient presented with a massive bilobar metastatic liver involvement, including S4. Five lesions were localized in a small left liver lobe (future liver remnant $<25 \%$ ). During the first stage of ALPPS, the liver parenchyma was transected with preservation of the central part of the middle hepatic vein, followed by a nonanatomical resection of S3 and a metastasectomy in S2. The procedure was completed by radiofrequency ablation of S2 lesions close to the S2 portobiliary triad, to spare venous drainage for S3. The second stage of ALPPS was performed 8 days later.

Results. Operative time was $300 \mathrm{~min}$ for the first stage of ALPPS and $200 \mathrm{~min}$ for the second stage. Peroperative blood loss did not exceed $50 \mathrm{~mL}$ per operation, and no postoperative complications were observed. The patient
\end{abstract}

Electronic supplementary material The online version of this article (https://doi.org/10.1245/s10434-018-6930-7) contains supplementary material, which is available to authorized users.

(C) Society of Surgical Oncology 2018

First Received: 21 July 2018;

Published Online: 29 October 2018

M. Smolár, $\mathrm{MD}, \mathrm{PhD}$

e-mail: smoly2002@hotmail.com was discharged 7 days after the second surgery. One month later, a laparoscopic uncomplicated low anterior resection with tumor-free resection margins was performed. Five months after surgery, no disease progression was detected. Conclusion. A laparoscopic ALPPS procedure with preservation of one portobiliary triad in the left lobe would be feasible in selected patients. The laparoscopic approach would be very important for patients waiting for a final primary tumor surgery.

DISCLOSURE None.

\section{REFERENCES}

1. Machado MAC, Makdissi FF, Surjan RC, Basseres T, Schadde E. Transition from open to laparoscopic ALPPS for patients with very small FLR: the initial experience. HPB (Oxford). 2017;19(1):59-66.

2. Fretland ÅA, Kazaryan AM, Bjørnbeth BA, et al. Open versus laparoscopic liver resection for colorectal liver metastases (the Oslo-CoMet study): study protocol for a randomized controlled trial. Trials. 2015;16:73. 